\title{
Linear and Non-Linear Companding Transformation Reducing PAPR in OFDM Signals
}

\author{
Sadu Venkata Naresh, P. V. Naganjaneyulu, P. Nageswara Rao
}

\begin{abstract}
An important disadvantage of Orthogonal Frequency Division Multiplexing (OFDM) signals is high peakto-average power ratio (PAPR), causing genuine debasement popular execution when a nonlinear power amplifier $(P A)$ is utilized. A notable technique to diminish power ratio deprived of confinements on framework parameters is Companding Transform (CT), for example, the sum of subcarriers, outline organization, and gathering type. Another nonlinear companding change, the utilization of hyperbolic transfer sigmoid function capacity is proposed for PAPR decrease in this paper. The proposed methodology extends the little flag and packs the pinnacle flag in the time zone at the same time without changing the average power. Results dependent on MATLAB demonstrate that the proposed methodology increases additional pinnacle decrease and improves the BER (bit error ratio)execution.
\end{abstract}

IndexTerms-Companding transform $(C T)$, non-linear, hyperbolic tangent sigmoid transfer function, OFDM, peak-toaverage power ratio $(P A P R)$.

\section{INTRODUCTION}

OFDM is cast-off for wide band wireless communication classifications like Worldwide Inter-operability for Microwave Access (WiMAX), Terrestrial Digital Video Broadcast (DVB-T), or wireline structures like Asymmetric Digital Subscriber Line (ADSL). The most extreme issue of the OFDM is the lopsided pace of the PAPR of the communicated signal. Because of the superposition of the various subcarriers, the frequency multiplexing signals show cases Rayleigh-like attributes. A huge variety realize over the top qualities for the power ratio. All points require High Power Amplifiers (HPA) to direct an inside and out straight unique assortment. A higher sign advance on the enter of HPA causes non-straight bends at its yield, prompting ineffective activity of HPA. These alterations process intermodulation items resultant in undesirable out-of-band control. To diminish the power ratio of frequency division signals, a few outcomes are anticipated then inspected. A portion of the real patterns of those procedures are nonlinearity, calculation complexity, length of angle insights that should have been dispatched to the beneficiary. A portion of the widely perceived straight techniques are Selective Mapping (SLM) [1], Partial Transmit Sequence (PTS) [2], and Tone Reservation (TR) [3]. Signal mutilation

Revised Manuscript Received on August 05, 2019.

Sadu Venkata Naresh, Research Scholar, JNTUH, Hyderabad, Telangana, India.

(E-mail: svenkatanaresh@gmail.com)

Dr. P. V. Naganjaneyulu, Principal \& Professor of ECE, SMCE, Guntur, A.P, India.

(E-mail pvnaganjaneyulu@gmail.com)

Dr. P. Nageswara Rao, Director \& Professor of ECE, MPES, Guntur, A.P, India

(E-mail: pnrao33@gmail.com) answers which incorporate cut-out [3][4][7] companding [5][6] might be utilized lacking limitation over device parameters anyway at the charge of delayed bit error rate (BER) and phantom re-development. A modified nonlinear companding transform with the aid of manner of the use of hyperbolic tangent sigmoid switch characteristic for PAPR reduction changed into proposed on this paper. It can restriction the sort of sign amplitude successfully with the aid of way of compacting huge indicators while enhancing small ones. If the parameters of the companding feature are selected properly, an exquisite PAPR discount can be gained and the BER performance is appropriate. The paper representation is printed such as Section II tends to the Peak power ratio of frequency multiplexing signaling cautions. Area III presents the projected hybrid transform, Area IV talks about output results. The paper is resolved in part V.

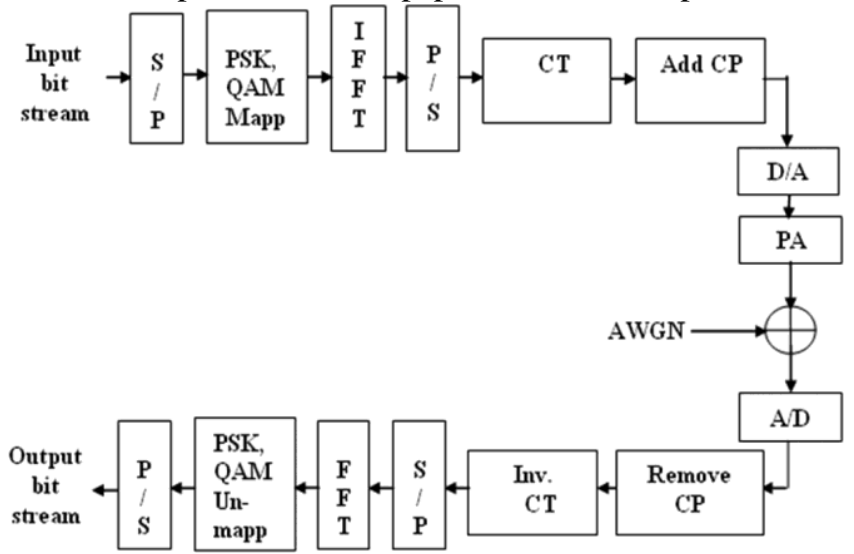

Fig. 1. Standard companded OFDM system.

\section{FORMULATION OF PAPR}

A standard companded system, in which entered bit movement is initially transformed into $\mathrm{N}$ equivalent minor price bitstreams and then fed into symbol plotting to attain signs $\left[S_{\mathrm{k}}=\mathrm{S}_{0}, \mathrm{~S}_{1} \ldots, \mathrm{S}_{\mathrm{N}-1}\right]$ was suggested in Fig. 1

These symbols, later used on IFFT to create OFDM symbol, expressed as

$$
x_{n}=\frac{1}{\sqrt{N}} \sum_{k=0}^{N-1} X_{k} e^{j 2 \Pi} \frac{n}{N} k, n=0,1,2 \ldots N-1(1)
$$

Where $j=\sqrt{ }-1$ and the PAPR of OFDM signal in (1) can be written as

$$
P A P R=\frac{\max \left|x_{n}\right|^{2}}{E\left[\left|x_{n}\right|^{2}\right]}
$$

where $E$ [.] denotes expectation 


\section{THE PROPOSED COMPANDING METHOD}

In this paper, we suggestan innovative procedure, that compresses the small signals and expands the large signals simultaneously by using hyperbolic tangent sigmoid transfer function. The model of OFDM transmitter and receiver adopting the projected method is shown in Figure.2.

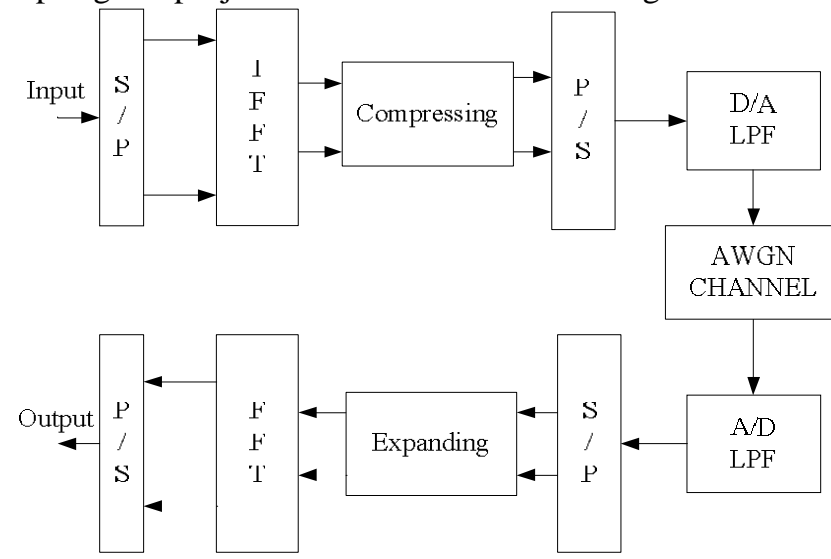

Fig.2 OFDM system adopting the companding method

The hyperbolic tangent sigmoid transfer function is given by

$$
\tanh (x)=\frac{e^{x}-e^{-x}}{e^{x}+e^{-x}}
$$

And the companding function for the new companding transform is defined as follow

$$
s_{n}^{\prime}=\frac{\alpha \times s_{n} \times V}{\left|s_{n}\right|} \times \tanh \left(\frac{k \times\left|s_{n}\right|}{V}\right)
$$

where $V$ is the value of the average amplitude of the OFDM signals and is almost constant. $\alpha$ and $k$ are the companding parameters. $k$ is chosen firstly and then $\alpha$ is determined corresponding to the chosen $k$ with the constraint that the average power is not changed by the companding transform for shifting OFDM peak power ratio. From (4), it is obviously observed $\left|S_{n}\right|$ that cannot be larger than $\alpha \times V$. And the proposed method compresses the large peak better than the Huang companding method.To maintain inter-block interference (IBI) cyclic prefixing (CP) code of span, NC which is basically the guarding time is attached in the front of each square after the companding movement. The time of $\mathrm{CP}$ code needs to not be not exactly the most length of the channel while a greater expense than greatest abundance put off of the channel is anticipated for limiting the IBI. Because of the ways of life of $\mathrm{CP}$ in the channel, the straight convolution system is by all accounts equivalent to the round convolution. To achieve the frequency area equalization technique possible inside the less than desirable end, the consideration of CP is practiced. At long last, a heartbeat molding clear beats the separating activity on the sign before being transmitted through a remote channel. In this way, the better PAPR execution can be normal by utilizing the proposed strategy.

In order to maintain the average power unchanged and to avoid the AWGN noise enhancement [6] on the receiver, the parameters $\alpha$ and $\mathrm{k}$ are selected with the limitations:

$$
E\left(s^{2}\right)=E\left(s^{\prime 2}\right)
$$

As the real and the imaginary part of $s_{n}$ is Gaussian distributed, $E\left(s^{2}\right)$ is almost constant. From [7], the true power of the companded signal can be given by

$$
E\left(s_{n}^{\prime 2}\right)=\int_{-\infty}^{\infty}\left[\frac{\alpha \times s_{n} \times V}{\left|s_{n}\right|} \tanh \left(\frac{k \times\left|s_{n}\right|}{V}\right)\right]^{2} f_{s_{n}}\left(s_{n}\right) d s_{n}
$$

(6)

Where $f_{S_{n}}\left(s_{n}\right)$ is the probability density function from (5) $\&$ (6) it is obvious that when $k$ is chosen, the other parameter $\alpha$ is also determined. At the receiver, the established signal after inverse-companding is given by

$$
R_{n}=0.5 \times \frac{V \prime \times R_{n}^{\prime}}{k \times\left|R_{n}^{\prime}\right|} \times \ln \left\{\frac{\alpha \times V \prime+\left|R_{n}^{\prime}\right|}{\alpha \times V \prime-\left|R_{n}^{\prime}\right|}\right\}
$$

Where $\mathrm{R}_{\mathrm{n}}{ }^{\prime}$ is the received signal and $V{ }^{\prime}$ is theaverage amplitude of the received signals. Because $\left|R_{n}\right|$ can not be larger than $\alpha \times V^{\prime}$, we can set a threshold for $\left|R_{n}{ }^{\prime}\right|$ which is a little less than $\alpha \times V^{\prime}$ to avoid the AWGN noise added to the peak signal.

\section{SIMULATION AND RESULTS}

To display the overall presentation of the projected nonlinear companding transform on decreasing PAPR and the gadget BER, we anticipate that randomly generated facts are modulated via 16QAM and the variety of the subcarriers is $\mathrm{N}=1024$ at some stage in simulation.

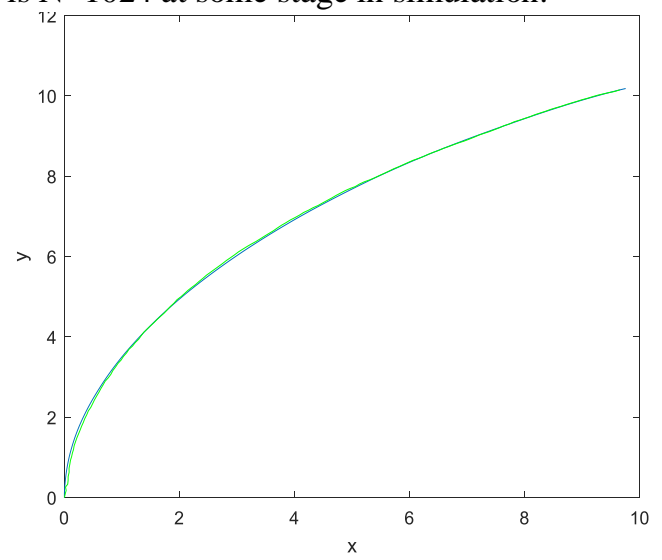

Fig.3 Linear Companding transform

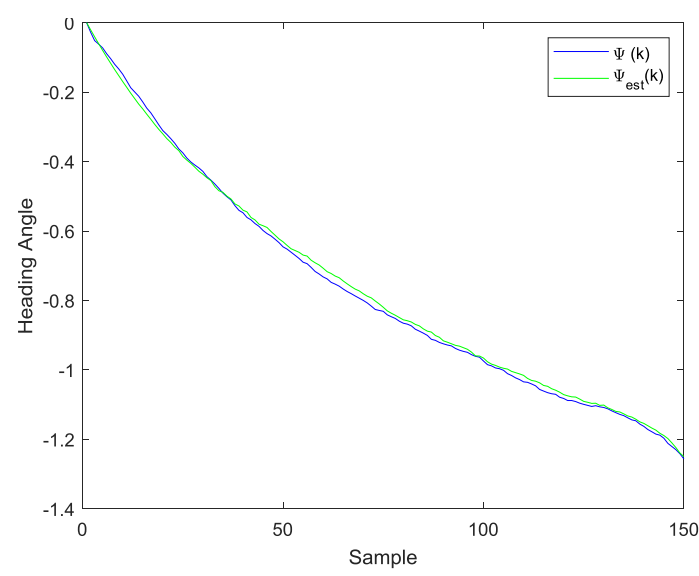

Fig.4 Nonlinear CompandingTransform 


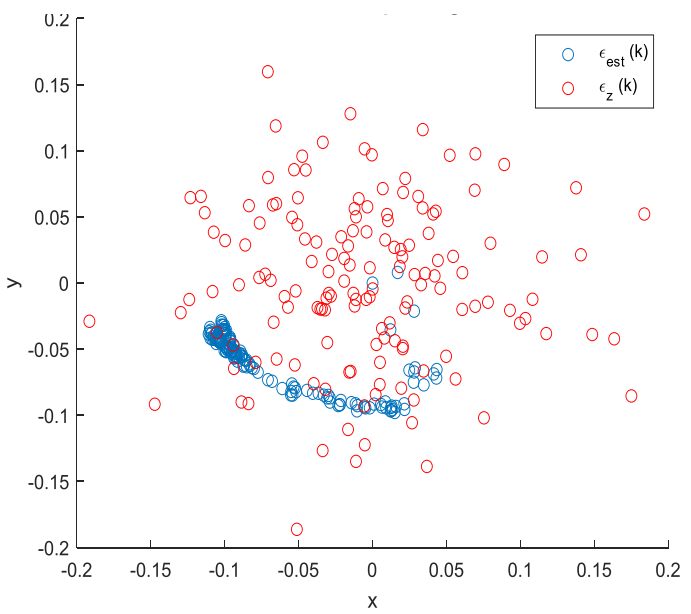

Fig.5 Linear and Non-Linear Companding Transform

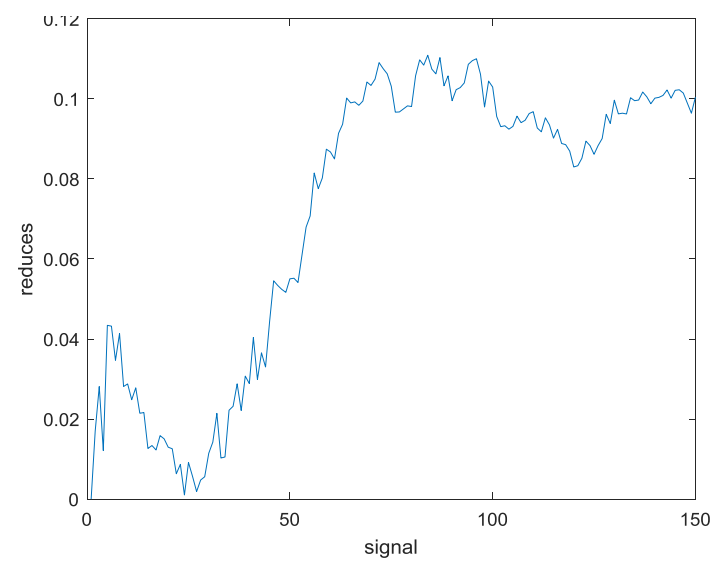

Fig.6 PAPR reduction performance

\section{CONCLUSION}

Linear and Nonlinear companding transform is a viable procedure in diminishing the PAPR of OFDM signals with the condition that the quantity of the sub-carrier is enormous enough $(\mathrm{N}>64)$. Also, the plans dependent on the companding method have low usage operation difficulty and absence of pre-requisite on modulation format and subtransporter size. This paper projects another companding transform by utilizing hyperbolic tangent sigmoid transfer function. This proposed companding change diminishes PAPR more proficiently and shows preferred BER execution over the earlier companding transforms. By expanding the estimation of the parameter $\mathrm{k}$, we can show signs of improvement PAPR execution for relinquishing the system execution a bit.

\section{REFERENCES}

$1 \quad$ S. Muller, R. Bauml, R. Fischer, and J. Huber, "OFDM with reduced height-to-common power ratio via multiple sign representation", Annals of Telecommunications, vol. Fifty three, pp. Fifty eight-67, February 1997.

2 L. J. Cimini Jr. And N. R. Sollenberger, "Peak-tocommon electricity ratio reduction of an OFDM sign the use of partial transmit sequences", IEEE Commun. Lett., vol. 4, no. Three, pp. 86-88, March 2000.

3 L. Wang and Y. Cao, "Improved SLM for PAPR Reduction in OFDM Systems", International Workshop on Intelligent Systems and Applications, pp. 1-four, May 2009.
4 J.Armstrong, "Peak-toaveragereductionforOFDMbyrepeatedclip- ping and frequency domain filtering," IEE Electron. Lett., vol. 38,pp. 246-247, May2002.

$5 \mathrm{X}$. Li and L. J. Cimini, Jr., "Effects of clipping and filtering at the in keeping with- formance of OFDM," IEEE Commun. Lett., vol. 2, no. Five, pp. 131-133, May 1998.

6 X. Wang et al., "Reduction of top-to-average strength ratio of OFDM system the usage of a companding method," IEEE Trans. Broadcast., vol. Forty five, no. Three, pp. 303-307, Sep. 1999.

7 S. A. Aburakhia, E. F. Badran, and D. A. E. Mohamed, "A assessment between signal clipping and -law companding schemes for the re- duction of peak-toaverage strength ratio of OFDM indicators," in Proc. 1st Int. Conf. On Digital Commun. \& Computer Applications DCCA2007, Irbid, Jordan, Mar. 2007, pp. 423-431.

8 S. C. Thompson, J. G. Proakis, and J. R. Zeidler, "The effectiveness of signal clipping for PAPR and total degradation reduction in OFDM systems," in Proc. Of the IEEE Global Telecommun. Conf., St. Louis, Dec. 2005, vol. Five, pp. 2807-2811. 